\title{
PINZAMIENTO ANTEROLATERAL DEL TOBILLO. EVALUACION ECOTOMOGRAFICA
}

\author{
Drs. Alonso Inzulza C, Winston Robertson C.
}

Clínica San José Arica.

\begin{abstract}
Ultrasound anatomic evaluation of normal and pathologic ligaments of the lateral aspect of ankle was performed. The objective of this study was to determine the usefulness of this exam in patients suggesting anterolateral impingement. Both sides were studied in fifteen (15) normal patients and in fourteen (14) patients submitted by the orthopedic surgeon with suggestive symptoms. Ligaments studied included: anterior tibiofibular, anterior talofibular and calcaneofibular. They were observed as an echogenic strip with posterior hypoechoic structures. Eleven (11) ligaments demonstrated alterations in eight (8) patients, with swelling and convexity of the strip. Four (4) patients had arthroscopic surgery and good correlation with ultrasound was found. Highresolution ultrasound can be a good diagnostic exam in anterolateral impingement especially if it is considered as an economic alternative when compared to $M R$.
\end{abstract}

Key words: Ultrasound, Ankle.

Resumen: Se realizó una evaluación de la anatomía ultrasonográfica de los ligamentos normales y patológicos de la región lateral del tobillo, con el objeto de determinar su utilidad en pacientes con clínica sugerente de pinzamiento anterolateral. Se estudiaron ambos lados en 15 pacientes sanos y en 14 pacientes referidos por traumatólogo con clínica sugerente. Los ligamentos evaluados fueron los: tibioperoneo anterior (TPA), peroneoastragalino (PA) $y$ peroneocalcáneo. (PC) En pacientes sanos se observan como bandas ecogénicas, rectas, con estructura hipoecoica posterior. En 8 pacientes con sospecha clínica se detectó alteración en 11 ligamentos, observándose aumento de volumen y convexidad de la banda. A cuatro de estos pacientes se les practicó cirugía endoscópica

Inzulza A, Robertson W. Pinzamiento anterolateral del tobillo. Evaluación ecotomográfica. Rev Chil Radiol 2002; 8: 28-32.

Correspondencia: Dr. Alonso Inzulza. Guillermo Sánchez 670 Dpto. 41-E, Arica. E-mail: alonsoandrea@entelchile.net encontrándose una buena correlación con los hallazgos ecotomográficos. En todos se indicó correctamente los ligamentos comprometidos.

La ecotomografía de alta resolución puede ser un buen método diagnóstico en el pinzamiento anterolateral del tobillo a nivel de PA y TPA, especialmente si se considera como método alternativo a la RM por su bajo costo y accesibilidad.

Palabras Claves: Ultrasonido, Tobillo.

\section{Introducción}

El pinzamiento anterolateral del tobillo (PAL) fue descrito por Wolin en 1950 y es producido por el anormal atrapamiento de partes blandas en la gotera anterolateral del tobillo. Los pacientes refieren dolor crónico, edema y limitación funcional. La mayoría de los casos tiene como principal antecedente la inversión reiterativa del tobillo. Después de estos episodios se estima que alrededor de un 10 a $50 \%$ de los pacientes presentan estos signos ${ }^{(1)}$.

La lesión mencionada puede estar formada por tejido sinovial hipertrofiado, cicatriz fibrosa o un fascículo accesorio del ligamento tibioperoneo anterior.

El diagnóstico diferencial es amplio, síntomas similares pueden ser atribuidos a inestabilidad crónica del tobillo, ruptura o subluxación de los tendones peroneos, fractura de estrés, lesiones osteocondrales, coalición tarsal, pinzamiento óseo, y enfermedad degenerativa articular.

La artroscopía confirma el diagnóstico y es el tratamiento definitivo al remover el tejido alterado.

La resonancia magnética (RM) es el método de elección en su estudio. El desarrollo de la artroresonancia ha mejorado los resultados ${ }^{(2-6)}$. En nuestro país la disponibilidad de esta técnica es aún limitada, principalmente en provincias lo que se traduce en falta de apoyo diagnóstico para los clínicos, ello nos motivó a estudiar la anatomía ecotomográfica de la región lateral del tobillo y determinar las características en pacientes con clínica sugerente del síndrome de PAL.

\section{Material y método}

Entre Noviembre de 2000 y Julio de 2001 se realizó un estudio prospectivo que consistió en 
ecotomografías bilaterales de tobillo en 15 pacientes sanos con el fin de determinar la anatomía habitual y en 14 pacientes referidos por traumatólogo con clínica sugerente de pinzamiento anterolateral.

Los estudios fueron realizados en la Clínica San José de Arica con ecotomógrafo ATL HDI 3500, usando transductor lineal de $5-12 \mathrm{MHz}$. La exploración fue realizada con cortes establecidos siguiendo la dirección longitudinal de los ligamentos tibioperoneo anterior (TPA), peroneoastragalino (PA) y peroneocalcáneo (PC) (Figura 1). Las edades fluctuaban entre los 18 y 50 años. En el grupo control se evaluó 8 mujeres y 7 hombres y en el grupo con clínica 8 y 6 respectivamente. Todos los pacientes tenían radiografías de tobillo en dos planos interpretadas como normales.

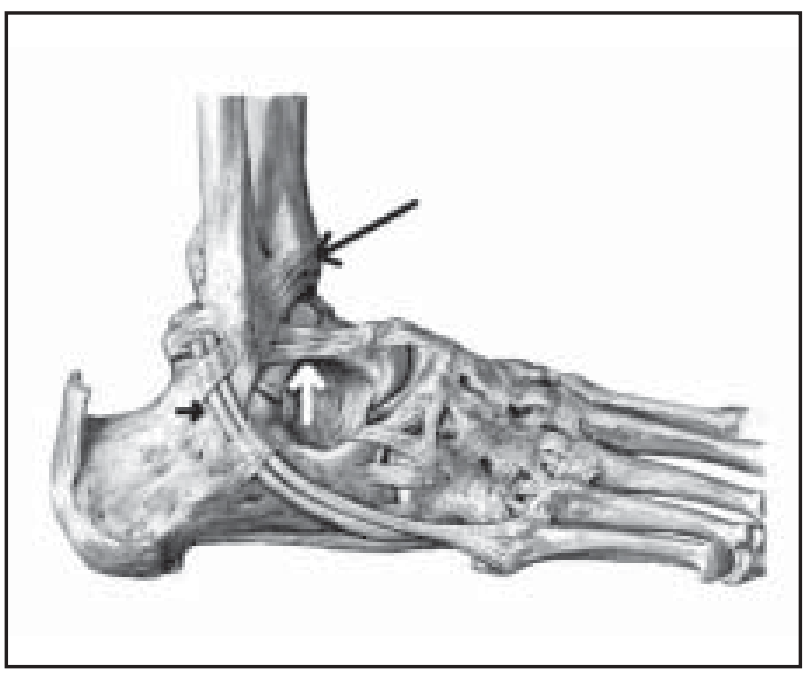

Figura 1. Anatomía de la región anterolateral del tobillo mostrando los ligamentos tibioperoneo anterior (flecha negra larga), peroneoastragalino (flecha blanca) y peroneocalcaneo (flecha negra corta).

(Modificado de referencia 9).

\section{Resultados}

En la evaluación del grupo control se observó los complejos ligamentosos PA y TPA como bandas hiperecogénicas, rectas, con estructura posterior hipoecogénica homogénea triangular (Figura 2). EI ligamento PC fue de más difícil visualización ya que su posición muestra una banda recta con estructura posterior mal definida lo que no permitió establecer un patrón constante.

Las características mencionadas mostraron total simetría en todos los casos estudiados.

En los 14 pacientes con clínica sugerente de síndrome de PAL se encontró alteración considerada como significativa en 8 , siendo siempre unilateral, en concordancia con la clínica. En un paciente se estimó como sector comprometido el del TPA, en cuatro el PA y en tres pacientes ambos. De esta forma se consideró compromiso en 11 sectores ligamentosos (Tabla I).

Los principales signos ecotomográficos fueron: Aumento de volumen posterior con convexidad de la banda principal, observado en todos los casos $(100 \%)$, hiperecogenicidad posterior que se observó en 7 de los sectores $(63,6 \%$ ) y alteración de la estructura (hetererogenicidad), que se apreció en 5 de dichos sectores $(45,5 \%)$ (Tabla II).

En algunos de nuestros casos se observó como signos asociados: mala definición o irregularidad de la banda principal, discontinuidad de ésta, presencia de una pequeña banda líquida y edema.

Se consideró asimetría una diferencia sobre un milímetro con relación a la banda ecogénica principal, al trazar una recta desde ambos segmentos óseos estudiados.

Cuatro pacientes fueron sometidos a cirugía artroscópica, apreciándose en ellos una masa de tejido fibroso con un componente menor de sinovitis y contenido hemático, depositado en los sectores ligamentosos indicados por la ecotomografía, realizándose resección. De estos pacientes 2 tenían alteración a nivel del PA, uno del TPA y uno en ambos (Figuras 3 y 4 ).

De los seis pacientes con clínica sugerente pero con examen ecotomográfico normal, solo tres fueron seguidos y controlados por traumatólogo, experimentando mejoría de sus síntomas con tratamiento médico.

Tabla I. Ligamentos alterados en 8 pacientes (11 ligamentos)

$\begin{array}{lll}\text { PA } & \text { 4 Pacientes } & \text { (4) } \\ \text { TPA } & \text { 1 Paciente } & \text { (1) } \\ \text { TPA+PA } & \text { 3 Pacientes } & \text { (6) }\end{array}$

( ) : ligamentos

Tabla II. Hallazgos ecográficos en 8 pacientes (11 ligamentos)

Aumento de volumen posterior (11) $(100,0 \%)$ Hiperecogenicidad posterior (7) (63,6\%) Alteración de la estructura (5) (45,5\%)

( ) : ligamentos 

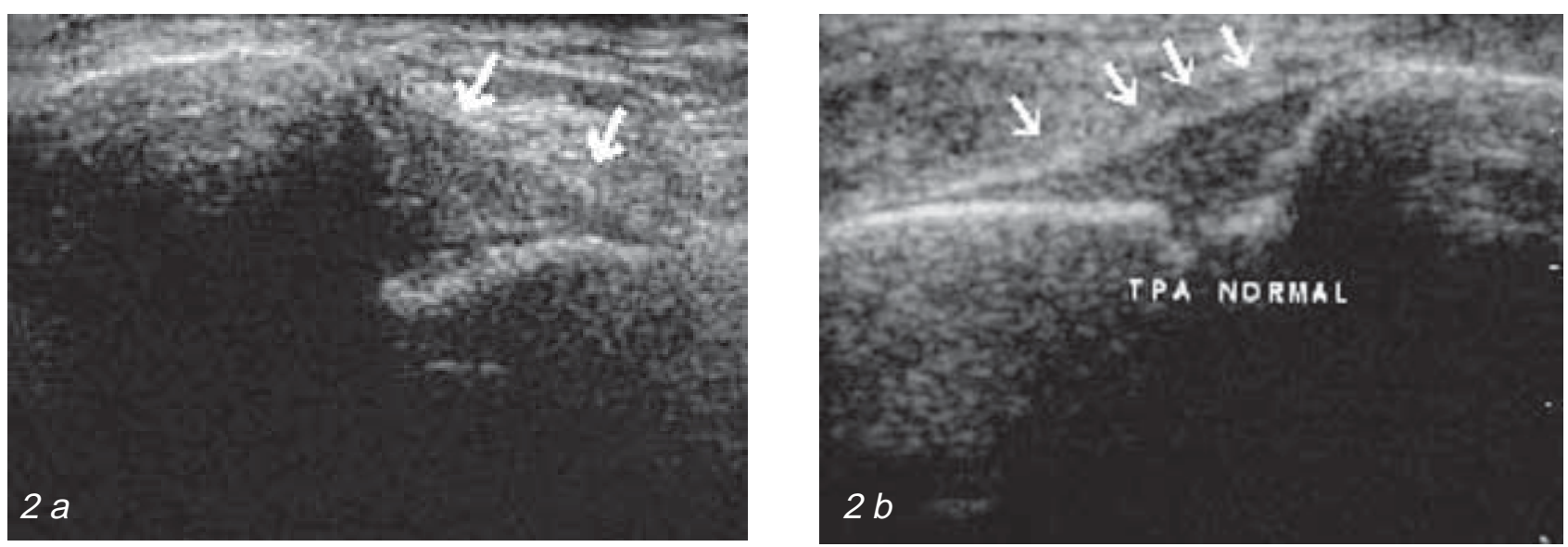

Figuras 2 a, b. Ecotomografías de ligamentos normales. a. Peroneoastragalino en mujer de 22 años (flechas). b. Tibioperoneoanterior en hombre de 35 años (flechas).
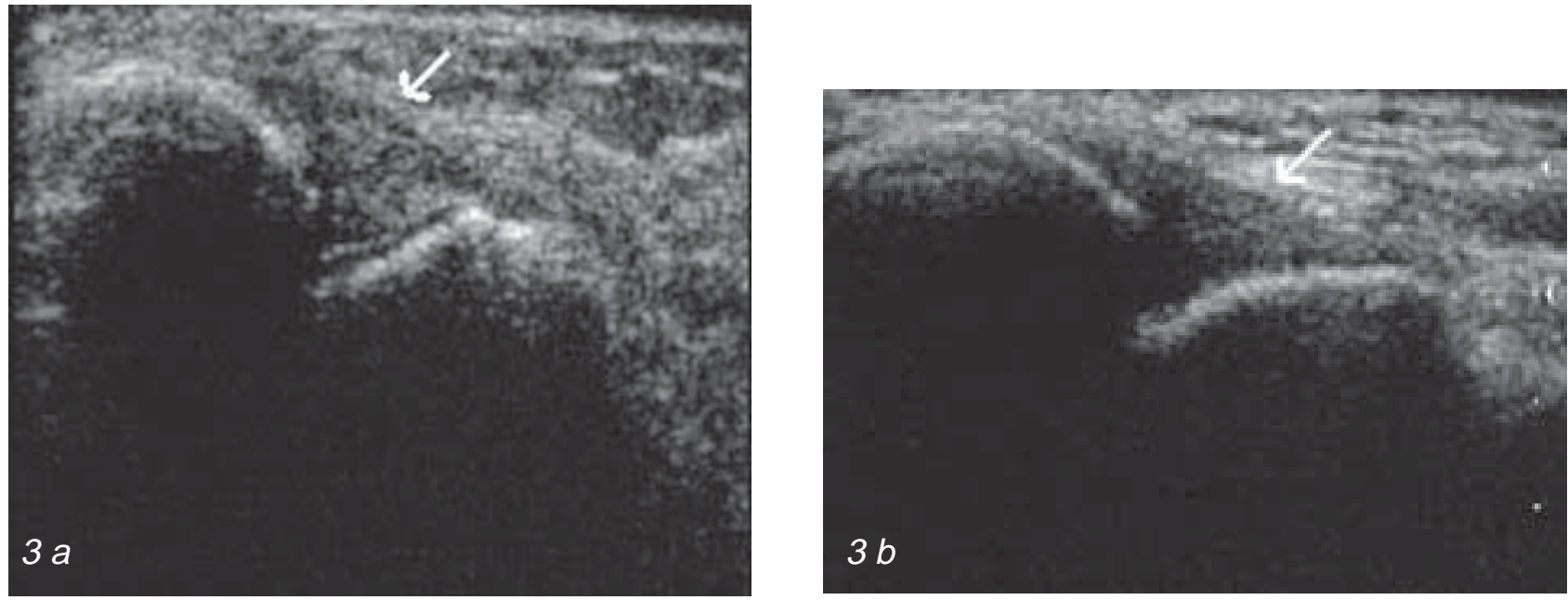

Figuras 3 a, b. Paciente de 46 años con compromiso del Peroneoastragalino (PA) derecho. a. PA derecho, hiperecogénico y aumentado de volumen con incurvación de la banda. Comprobación artroscópica. b. Lado izquierdo PA normal (flecha).
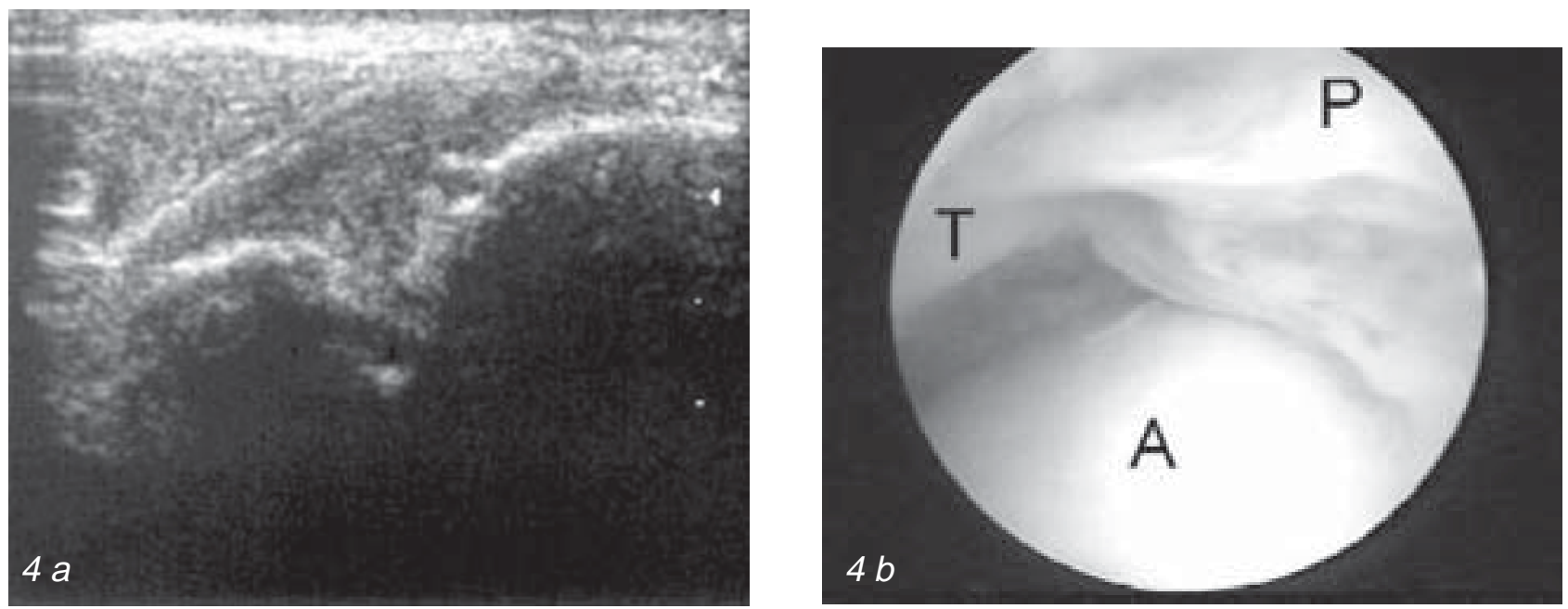

Figuras 4 a, b. Paciente de 46 años con pinzamiento de TPA izquierdo. a. Ultrasonografía demuestra aumento de volumen, hipercogenicidad y alteración de la estructura posterior. b. Artroscopía confirmando los hallazgos T: Tibia, P: Peroné, A: Astrágalo. 


\section{Discusión}

El síndrome de PAL del tobillo fue descrito primero por Wolin, al apreciar que en nueve pacientes con antecedentes de dolor persistente y edema del tobillo, luego de inversiones repetidas, la cirugía mostraba una masa de partes blandas depositada en la gotera anterolateral del tobillo a la que denominaron lesión "meniscoidea". Los autores sugirieron que la inversión del tobillo producía una sinovitis traumática asociada a hemorragia. Más tarde otros investigadores han sugerido que la inflamación de los ligamentos dañados contribuye a la formación de la masa ${ }^{(1)}$.

El síndrome ha sido atribuido también a un fascículo accesorio de TPA, esta variante normal es una estructura intra-articular que se extiende desde la epífisis distal anterolateral de la tibia a la epífisis distal anteromedial del peroné. Este fascículo accesorio después de repetidos traumas produce pinzamiento y es clínicamente indistinguible del pinzamiento causado por sinovitis o tejido cicatricial ${ }^{(1)}$. Dicha variante no fue apreciada en los casos comprobados de nuestro estudio.

Los pacientes con pinzamiento presentan dolor anterolateral del tobillo principalmente con la actividad física lo que limita la práctica deportiva. Otros signos incluyen exacerbación del dolor con la dorsiflexion y efusión.

La alteración compromete principalmente al ligamento PA ya que este sector representa un punto débil, así como también, el ligamento TPA del complejo ligamentoso sindesmal. Los principales trabajos encontrados en la literatura, todos con RM atribuyen al complejo ligamentoso del PA la casi exclusiva incidencia ${ }^{(2-6)}$.

El tratamiento es inicialmente conservador con una combinación de inmovilización, anti-inflamatorios no esteroidales, terapia física o inyección de esteroides. Los pacientes que no responden a este tratamiento se benefician con la artroscopía a fin de identificar y resecar el tejido.

Rubin y col. ${ }^{(2)}$ en un estudió retrospectivo de 18 pacientes con confirmación artroscópica de pinzamiento anterolateral, demostraron 9 casos con líquido, de los cuales ocho (89\%) presentaban masa de partes blandas en dicha región. Fue imposible distinguir pinzamiento producido por ligamento TPA accesorio del producido por tejido cicatricial o sinovitis. Concluyen que este diagnóstico puede ser sugerido por RM solo ante la presencia de líquido en la gotera anterolateral.

Liu y col. ${ }^{(3)}$ evaluaron 22 pacientes con RM y artroscopía. La RM tuvo una sensibilidad de 39\% y especificidad de $50 \%$ para predecir correctamente el pinzamiento versus $94 \%$ y $75 \%$ respectivamente del examen clínico. No recomiendan la RM ante la sospecha de síndrome de PAL e indican incluso que su realización puede retrasar el tratamiento.

Farooki, y col. ${ }^{(4)}$ publicaron un estudio en donde evalúan 12 pacientes con pinzamiento probado por artroscopía y 19 pacientes como grupo control demostrando una sensibilidad de un $42 \%$ y una especificidad de (85\%) para la RM indicando que el engrosamiento del PA y la presencia de masa de partes blandas en la gotera lateral puede sugerir este diagnóstico.

Bagnolesi y col. ${ }^{(5)}$ publican un trabajo en el cual estudiaron 14 pacientes en quienes se realizó $\mathrm{Rx}$, US, RM y artroscopia. La RM demostró una pequeña masa de tejido sinovial en la gotera en 8 pacientes y hallazgos normales en 6. La artroscopía mostró una pequeña masa firme de proliferación sinovial en la gotera lateral de todos los pacientes. Ellos tuvieron malos resultados con la ecotomografía y concluyen que es poco segura en el diagnóstico de esta condición y que la RM muestra una pequeña masa sinovial en solo la mitad de los pacientes e indican que debería ser efectuada con el uso de contraste.

Jordan y col. ${ }^{(6)}$ publicaron un estudio de 9 pacientes en los cuales la RM mostró en un $100 \%$ una masa de partes blandas e hipertrofia sinovial que fue comprobada artroscópicamente. El grupo control fue de 20 pacientes. Encontraron disrupción, atenuación o marcado engrosamiento del PA en todos los casos y concluyen que la RM en conjunto a una buena historia clínica pueden ser usadas para la indicación de resección artroscópica.

Hauger y col. ${ }^{(7)}$ publican un estudio con CT artrografía en 44 pacientes identificando cuatro patrones. Encontraron una excelente correlación con los hallazgos artroscópicos en los patrones tipos II y III descritos por ellos.

$S k^{(8)}$ señala que en su experiencia la realización de artroresonancia tiene una mejor certeza diagnóstica y mejora la visualización del PA.

De los datos publicados se puede establecer que la resonancia magnética es un buen examen en la evaluación de estos pacientes, aun cuando existen algunos estudios ${ }^{(3)}$ que al compararla con la sospecha clínica demuestran una baja especificidad y sensibilidad en el diagnóstico de esta condición, lo cual no avalaría su uso, al realizar una evaluación costo-beneficio. No conocemos un estudio local sobre esta problemática.

La ecotomografía en nuestra experiencia demostró correctamente las regiones alteradas lo que fue corroborado con artroscopía. en algunos de nuestros pacientes. Pese a que no se puede establecer una correlación exacta entre los signos ecotomográficos y los hallazgos operatorios, puede atribuirse al tejido fibroso la hiperecogenicidad visualizada en los complejos ligamentosos y al componente variable de éste y de sinovitis con contenido hemático, la alteración de la estructura y aumento de 
volumen. No fue posible una evaluación correcta del $\mathrm{PC}$ el que tampoco ha demostrado ser bien evaluado mediante otras técnicas de diagnóstico por imágenes, además la frecuencia de compromiso de él es muy baja según lo reportado en la literatura.

\section{Conclusiones}

La ecotomografía de alta resolución parece ser un buen método de diagnóstico en el pinzamiento anterolateral del tobillo cuando están comprometidos el PA y el TPA. No permite una buena aproximación diagnóstica cuando la alteración está dada a nivel del PC.

El bajo costo y disponibilidad de la ecotomografía y los resultados preliminares encontrados nos permiten sugerir su realización en pacientes con clínica sugerente de PAL, particularmente en lugares donde no se disponga o por costo no sea factible la realización de RM.

A pesar que nuestros casos hubo una buena correlación imagen-hallazgo artroscópico, se requiere una mayor cantidad de exámenes para determinar su sensibilidad y especificidad en el diagnóstico de pinzamiento anterolateral del tobillo.

\section{Bibliografía}

1. Ferkel RD. The foot and ankle. Arthroscopic Surgery. Lippincott Raven 1996.

2. Rubin DA, Tisshkoff NW, Britton CA y col. Anterolateral softtissue impingement in the ankle: diagnosis using MR imaging. AJR 1997; 169: 829-35.

3. Liu SH, Nuccion SL, Finerman G. Diagnosis of anterolateral ankle impingement. Comparison between imaging and clinical examination. Am J Sports Med 1997; 25: 389-93.

4. Farooki S, Yao L, Seeger LL. Anterolateral impingement of the ankle: effectiveness of MR imaging. Radiology 1998; 207: 357-60.

5. Balognesi P, Zampa V, Carafoli D, Cilotti A, Bartolozzi C. Anterolateral fibrous impingement of the ankle. Report of 14 cases. Radiol Med (Torino) 1998; 95: 293-297.

6. Jordan LK, Helms CA, Cooperman AE, Speer KP. Magnetic resonance imaging findings in the anterolateral impingement of the ankle. Skeletal Radiol 2000; 29: 34-39.

7. Hauger $\mathrm{O}$, Moinard M, Lasalarie JC y col. Anterolateral compartment of the ankle in the lateral impingement syndrome: appearance on CT arthrography. AJR 1999;173: 685-90.

8. Skib RA. Anterolateral soft tissue impingement of the ankle. Radiology 1998; 209: 885.

9. Netter FH. Atlas de Anatomía Humana. Masson S.A. 1996; pag 495. New Yersey. USA.

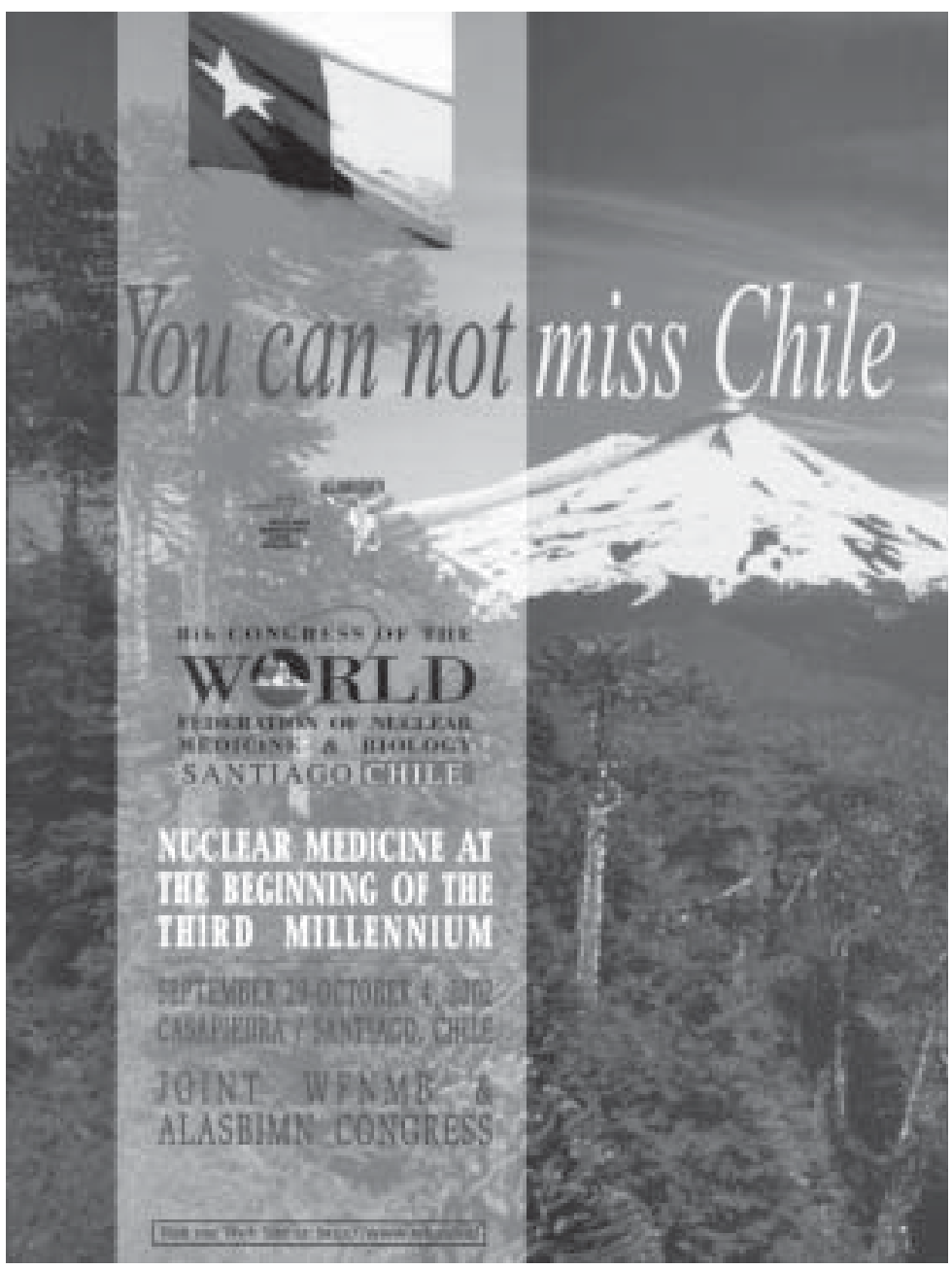

